\title{
RECURSOS DIDÁCTICOS 3D PARA EL APRENDIZAJE SIGNIFICATIVO DE ESTUDIANTES CON DISCAPACIDAD VISUAL
}

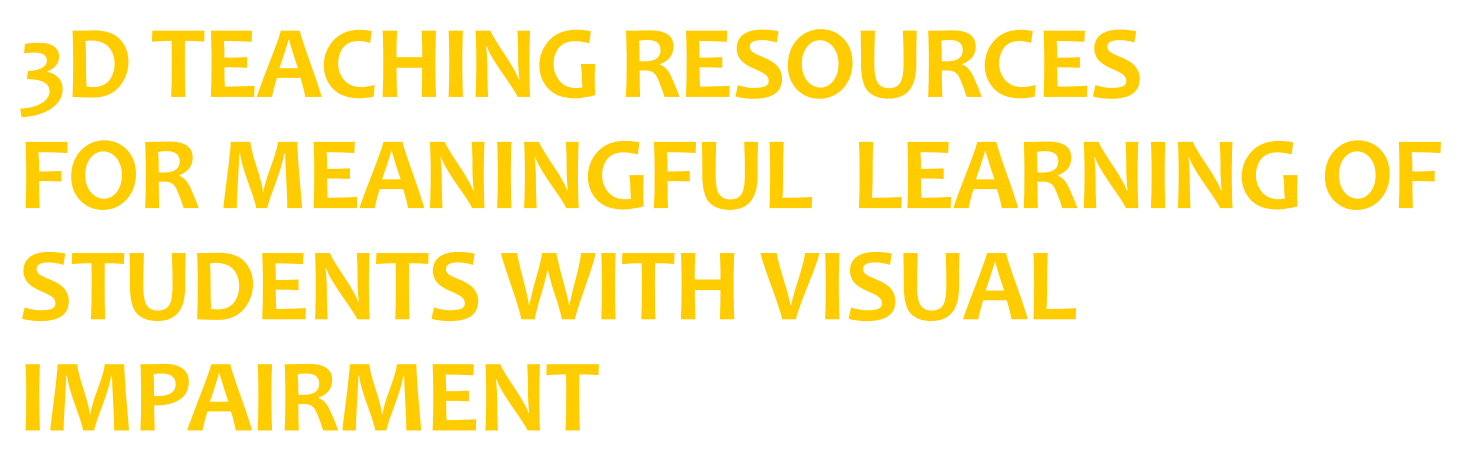

\section{Bertha Alice Naranjo Sánchez ${ }^{1}$}

\section{Danny Javier Banchón Morán²}

\section{Carlos Alberto Martínez Briones ${ }^{3}$}

Universidad Politécnica Salesiana Sede

Guayaquil

\footnotetext{
$1 \quad$ Bertha Alice Naranjo Sanchez. Docente e Investigadora de la Universidad Politécnica Salesiana Sede Guayaquil https://orcid.org/0000-0002-4386-2335 bnaranjo@ups.edu.ec

2 Estudiante Investigador de la Universidad Politécnica Salesiana Sede Guayaquil

dbanchonm @est.ups.edu.ec https://orcid.org/0000-0003-3765-9733

3 Docente e Investigador de la Universidad Politécnica Salesiana Sede Guayaquil cmartinezb@ups.edu.ec ttps://orcid.org/0000-0003-4136-1833
}

\section{RESUMEN}

En el Ecuador en el año 2019, el porcentaje de la población con discapacidad visual fue del $11.79 \%$, de ese porcentaje sólo el $4.51 \%$ eran niños que transitan la edad escolar y deben ser incluidos 
en la educación regular sin embargo muchos deben asistir a escuelas especializadas puesto que no todas las instituciones están preparadas para incluirlos. La tecnología, los recursos didácticos, entre otros constituyen algunos de los elementos necesarios para incluir a niños con discapacidad visual en el aula, puesto que ellos desarrollan un proceso de aprendizaje basado en otros sentidos, en especial el tacto, con el cual pueden percibir objetos o formas en general. Este artículo permitió analizar si los recursos 3D favorecen el aprendizaje significativo en los estudiantes con discapacidad visual. Para ello se desarrollaron recursos a medida, en una institución educativa especial y se aplicó como técnica la encuesta dirigida tanto a docentes como estudiantes, una entrevista a la directora de la institución y se obtuvo como resultado que los recursos impresos en tecnología 3D influyeron en el aprendizaje significativo de los estudiantes.

PALABRAS CLAVE: Tecnología 3D, Educación inclusiva, Fabricación aditiva, TIC, Aprendizaje significativo.

\section{ABSTRACT}

In Ecuador in the year 2019, the percentage of the population with visual impairment was $11.79 \%$, of that percentage only $4.51 \%$ were children transiting school age and should be included in regular education however many must attend specialized schools since not all institutions are prepared to include them. Technology, teaching resources, among others, constitute some of the elements necessary to include children with visual disabilities in the classroom, since they develop a learning process based on other senses, especially touch, with which they can perceive objects or forms in general. This article allowed us to analyze whether $3 \mathrm{D}$ resources favor significant learning in students with visual disabilities. For this purpose, customized resources were developed in a special educational institution and the survey aimed at both teachers and students, an interview with the director of the institution was applied as a technique and it was obtained as a result that resources printed in $3 \mathrm{~d}$ technology influenced the significant student learning.

KEYWORDS: 3D Technology, Inclusive education, Additive manufacturing, ICT, Meaningful learning.

\section{INTRODUCCIÓN}

Los recursos didácticos que usan los docentes pueden ser creados o adquiridos y en ambos casos se requiere de recursos económicos para su elaboración además de creatividad e imaginación. Pero la creatividad que tienen los docentes permite que los recursos que se desarrollen se acoplen a diversos presupuestos por ello encontramos recursos hechos con fomix, cartulinas, materiales reciclables, entre otros, sin embargo la durabilidad del recurso se ve afectada por el uso del mismo y su deterioro obliga que se vuelvan a hacer cada vez los mismos recursos, pero nos encontramos en la era de la educación 4.0 donde las tecnologías pueden y deben ser usadas en el aula para generar ese cambio y esa innovación que son necesarias para romper el enfoque de la clase tradicional.

Por ello proponemos el uso de productos provenientes de la industria 4.0 y uno de sus pilares que es la fabricación aditiva, en particular la impresión en tecnología 3D.

Este artículo explora el uso de tecnologías 3D, en particular de los recursos didácticos en $3 D$ con el fin de determinar si generan un aprendizaje significativo en los estudiantes con discapacidad visual. 


\section{MARCO TEÓRICO}

\section{Educación Inclusiva}

La Educación Inclusiva debe ser concebida como un proceso que permite abordar y responder a las necesidades de todos los estudiantes a través de una mayor participación en el aprendizaje y reducir la exclusión del sistema educativo. Esto implica cambios y modificaciones de contenidos, enfoques, estructuras y estrategias basados en una visión común y la convicción de que es responsabilidad del Sistema Educativo educar a todos los niños, niñas y adolescentes. El propósito de la Educación Inclusiva es permitir que los docentes y estudiantes se sientan cómodos ante la diversidad y la perciban no como un problema, sino como un desafío y una oportunidad para enriquecer el entorno de aprendizaje (Ministerio de Educación del Ecuador, 2018).

La educación inclusiva se preocupa de entender la manera como las personas funcionan, participan en las clases y cuáles son los obstáculos que enfrentan día a día en su proceso de aprendizaje (Tomalá Cabezas \& Naranjo Sánchez, 2019).

El articulo 28 capítulo cuarto: art. 10 en el inciso i, de la Ley Orgánica de Discapacidades del Ecuador, establece que: "...las instituciones educativas deben promover la inclusión de estudiantes con necesidades educativas especiales que requieran apoyos técnicos, tecnológicos, humanos, tales como personal especializado, temporales o permanentes y los docentes deben dar apoyo y seguimiento pedagógico a las y los estudiantes, para superar el rezago y dificultades en aprendizajes y en el desarrollo de competencias, capacidades, habilidades y destreza" (República del Ecuador, 2012).

Lo que propone la ley exige inversión en TIC (Tecnologías dela Informaciónyla Comunicación) y algunas instituciones educativas no cuentan con los recursos económicos suficientes para ello, pero este cambio es necesario para hacer realidad la inclusión.

En el Ecuador, en los últimos años “...se ha logrado la implementación de políticas inclusivas tras una larga lucha en un país donde antes se excluía a las personas con discapacidad" (Tomalá Cabezas \& Naranjo Sánchez, 2019). A pesar de que estas políticas de inclusión no tienen muchos años en vigencia, varios centros educativos han acatado estas leyes.

El artículo 47: numeral 7 sobre la igualdad y la inclusión precisa, “...poner en práctica procesos inclusivos tales como: una educación que desarrolle las potencialidades y habilidades de EcD (Estudiantes con discapacidad) para su integración y participación en igualdad de condiciones" (República del Ecuador, 2018).

Para brindar esa igualdad de condiciones los recursos didácticos que se diseñen para los estudiantes deben ser inclusivos o adaptados, ajustarse al contenido, objetivos o situaciones previas que inciten a la reflexión, la motivación y el desarrollo del pensamiento crítico, de esa forma se podrá crear un aprendizaje significativo en un entorno activo y participativo, donde el estudiante es el centro del proceso, tal como se aprecia en la tabla 1. 
Tabla 1: Estilos de aprendizaje.

\begin{tabular}{|l|l|l|l|}
\hline Activo & Reflexivo & Teórico & Pragmático \\
\hline Experiencias nuevas & Observadores & Lógicos y racionales & Prácticos \\
\hline Disfrutan el presente & Analíticos & $\begin{array}{l}\text { Integran las } \\
\text { observaciones en } \\
\text { teorías complejas y } \\
\text { coherentes }\end{array}$ & Realistas \\
\hline $\begin{array}{l}\text { Entusiastas ante lo } \\
\text { Nuevo }\end{array}$ & Precavidos & $\begin{array}{l}\text { Piensan en forma } \\
\text { secuencial }\end{array}$ & $\begin{array}{l}\text { Decisiones } \\
\text { rápidas en la } \\
\text { resolución de } \\
\text { problemas. }\end{array}$ \\
\hline $\begin{array}{l}\text { Rodeados de gente, } \\
\text { pero él es el centro de } \\
\text { las actividades }\end{array}$ & $\begin{array}{l}\text { Arriban a conclusiones } \\
\text { después de análisis } \\
\text { detallado }\end{array}$ & $\begin{array}{l}\text { Ajenos a los juicios } \\
\text { subjetivos }\end{array}$ & $\begin{array}{l}\text { Se impacientan } \\
\text { con las } \\
\text { discusiones } \\
\text { extensas sobre } \\
\text { una misma } \\
\text { cuestión }\end{array}$ \\
\hline
\end{tabular}

Fuente: (Gamboa, Briceño, \& Camacho, 2015)

Existen diversos tipos de discapacidades, este estudio se centra en la discapacidad visual. Los tipos de inteligencia más desarrolladas en niños con discapacidad visual son: la inteligencia representativa, la cual se evidencia con “...la capacidad de imitación en ausencia del modelo, en la exteriorización de las imágenes mentales mediante el dibujo, en la utilización de símbolos en el juego y en la posibilidad de comunicarse con los demás <<representados>> la realidad mediante el lenguaje" (Universidad de Las Palmas de Gran Canaria), tal como se observa en la tabla 2. Otro tipo de inteligencia que es muy característica en ellos es la inteligencia espacial, ya que, gracias a este tipo de inteligencia, ellos pueden reconocer ciertas formas a través de un método indirecto, pasar la mano a lo largo de un objeto (Proyecto Malala - Inteligencia VisualEspacial).

Tabla 2: Tipos de inteligencia más desarrolladas por personas con discapacidad visual

\begin{tabular}{|l|l|}
\hline Tipos de inteligencia & Conceptualización \\
\hline Inteligencia espacial & $\begin{array}{l}\text { Esta inteligencia la tienen profesiones tan diversas como la } \\
\text { ingeniería, la cirugía, la escultura, la marina, la arquitectura, } \\
\text { el diseño y la decoración. }\end{array}$ \\
\hline $\begin{array}{l}\text { Inteligencia } \\
\text { representativa }\end{array}$ & Imitación de un objeto físico mediante imágenes mentales. \\
\hline
\end{tabular}

Fuente: Elaboración propia, basado en (Mercadé) 
Los recursos didácticos le permiten materializar conceptos e ideas al estudiante. El docente aplicando los ajustes razonables puede lograr que todos los estudiantes desarrollen un aprendizaje inclusivo.

\section{TIC.}

Tradicionalmente, el estudio y la enseñanza están limitadas a la unilateralidad de la información y a la bidimensionalidad del papel o, en escenarios más modernos, a la de una pantalla, olvidando que en gran parte de las temáticas tratadas son representaciones de eventos y elementos que pertenecen a un universo tridimensional (Rúa, Blasnilo, Jiménez D, Gutiérrez A., \& Villamizar, 2018).

En (Arnaiz, 2012) se afirma que la escuela del siglo XXI debe promover una educación democrática e inclusiva que garantice los principios de igualdad, equidad y justicia social para todos los alumnos. Bajo el prisma de la escuela inclusiva, el uso de las TIC favorece el uso de elementos multimedia e interactivos que juegan un poderoso papel en la individualización de la enseñanza, generando así una presentación de los contenidos de forma dinámica, atractiva y personalizada. Es decir que mediante el uso de las TIC se puede innovar en la forma de presentar un tema y volver la clase dinámica, participativa, atractiva y personalizada, que capte la atención y motivación de los estudiantes.

\section{TECNOLOGÍAS EMERGENTES}

Las tecnologías emergentes transformarán las formas de pensar y de actuar con TIC destacando: a) Los recursos tecnológicos para la educación en general, b) Los recursos para la atención a la diversidad de manera especial, c) Las nuevas formas de promover y garantizar la igualdad de oportunidades, d) Los nuevos métodos de comunicación, e) Los novedosos marcos de referencia en la relación usuarioprofesional, f) las guías de proceso de valoración (Castro, Casar, \& García, 2018).
El informe Horizon4 entiende por tecnología emergente aquellas herramientas informáticas clave que, además de utilizarse hoy, prometen un crecimiento importante en los próximos años (Horizon Report, 2016). La impresión 3D es una tecnología emergente que seguirá presentando diversas formas de uso y aplicaciones en infinidades de áreas como la educación, cuyo campo aún falta explorar.

\section{TECNOLOGÍA 3D.}

La tecnología 3D también conocida como tecnología de la adición, según (Madrid, 2017), consiste en la creación de un objeto tridimensional mediante la superposición de capas sucesivas de material. Este tipo de tecnología es un proceso mediante el cual los datos de diseño digital en 3D se utilizan para construir objetos de casi cualquier forma y geometría en capas mediante el depósito de materiales (Umair \& Kim, 2015). A través del uso de esta tecnología, podemos llevar un diseño $3 \mathrm{D}$ a algo que se pueda ver y palpar.

Otro término empleado para la tecnología 3D es el de fabricación aditiva, la cual funciona incorporando el diseño de un objeto, su forma, a un modelo de computadora, y luego conduciendo ese modelo a capas separadas que pueden apilarse sobre otra para formar el objeto final (Horne \& Hausman, 2014), dando como resultado un producto en 3 dimensiones.

La tecnología 3D se ubica en lo más alto de la curva de tendencias como se muestra en la Fig. 1 , según la compañía de investigación Gartner. 
Figura 1. Curva Gartner 2019.

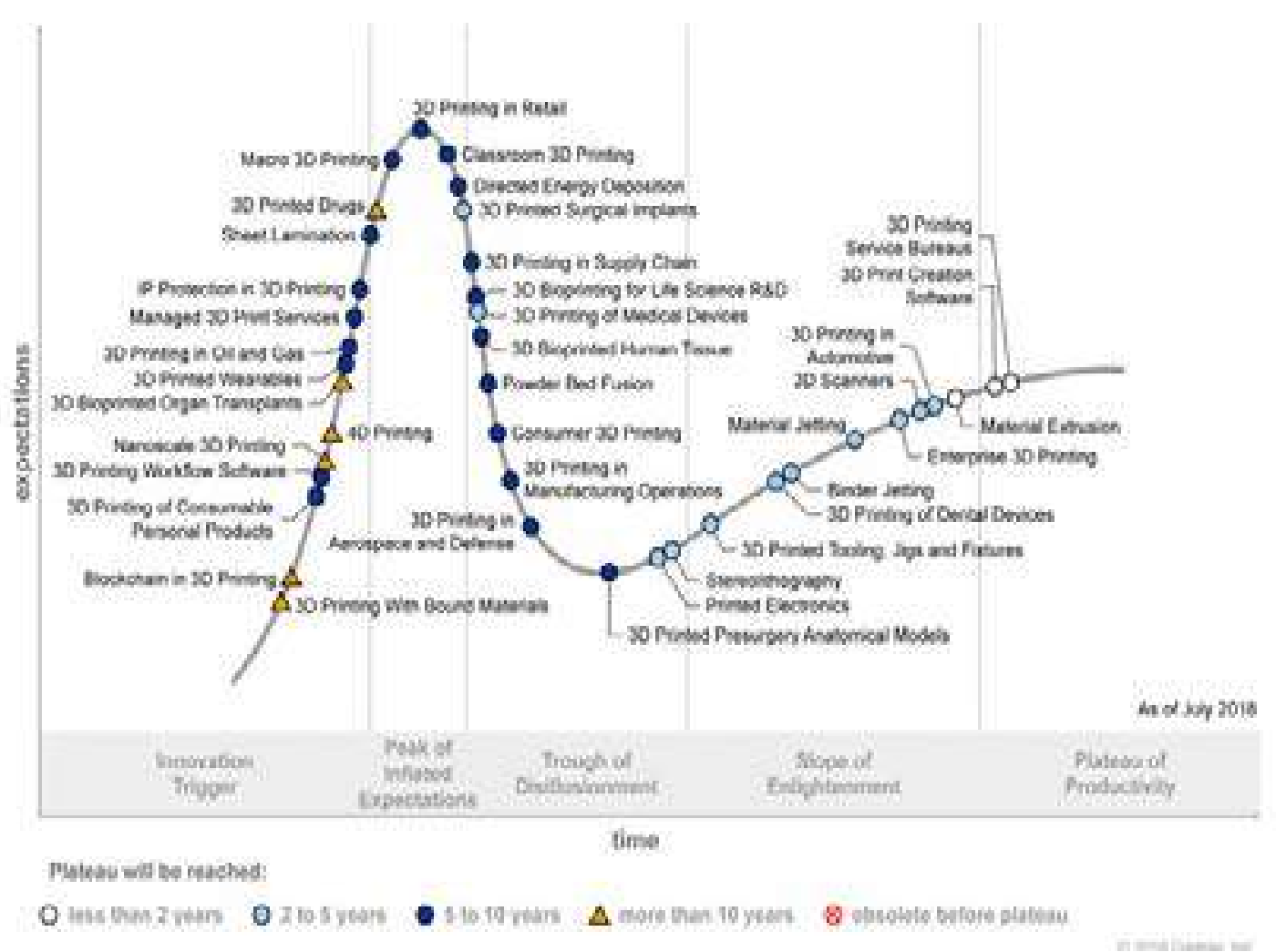

Fuente: Gartner citado en (Lucía, 2019)

Así los recursos didácticos en tecnología $3 \mathrm{D}$ podrían ayudar tanto a docentes como a estudiantes con discapacidad visual, a mantener un Proceso de Enseñanza y Aprendizaje (PEA) inclusivo. Los docentes podrán mejorar sus metodologías, técnicas de enseñanza, servicios y detectar problemas de aprendizaje que presenten los estudiantes (República del Ecuador, 2012).

\section{SISTEMA BRAILLE}

El sistema braille fue creado por Louis Braille, en 1824 el cual consta de un sistema de puntos en relieve de la lectura y la escritura para las personas ciegas y con deficiencia visual. EI braille es un sistema que permite a las personas ciegas o con discapacidad visual leer cualquier texto usando sus dedos (Sutariya, y otros, 2017). Las personas con discapacidad visual (PCDV) hacen uso de este sistema en la lecto-escritura de documentos, ellos poseen la capacidad de entender cuál es el significado de cada punto con relieve que se encuentre en los libros.

A pesar de que las personas con discapacidad visual acceden a los materiales de impresión mediante el uso de libros de audio o escuchar a un lector personal y escribir dictando a alguien, muchos encuentran que pueden acceder a la información más rápidamente y realizar tareas que implican la lectura o la escritura de manera más eficiente con el uso del Braille (Kway, Mohd Salleh, \& Abd. Majid, 2010). El sistema braille les permite desenvolverse de manera autónoma, deslizando las yemas de los dedos sobre matrices, determinando los símbolos para formar las palabras, siendo además un método alternativo de lectura para PCDV.

El diseño de recursos 3D junto al sistema braille aplicado como innovación educativa, pueden ayudar a favorecer el aprendizaje significativo de estudiantes con discapacidad visual. 


\section{APRENDIZAJE SIGNIFICATIVO}

Para Ausubel (citado en (Salguero Barba \& García, 2017)) el aprendizaje significativo es .... "un proceso por medio del cual se relaciona nueva información con algún aspecto, ya que existe en la estructura cognitiva de un individuo, debe ser relevante para el material que se intenta aprender".

Según (Chancusig Chisag, y otros, 2017) el aprendizaje significativo, es un proceso a través del cual el estudiante construye los nuevos conocimientos a base de las experiencias previas, siempre que encuentre sentido en lo que quiera aprender y tenga insuficiencia, interés y predisposición para ello, utilizando adecuadamente los recursos didácticos y está sujeto a una evaluación abierta y flexible.

Como destaca en (Cruz Verdugo, 2016)., "es importante que los docentes conozcan estrategias de aula para su aplicación y logro (...) procurar la utilización de materiales concretos, que afiancen el aprendizaje para hacerlo significativo".

El aprendizaje significativo permite que el estudiante, a través del uso de recursos didácticos proporcionados por el docente en el aula de clase, pueda afianzar o mejorar el proceso de obtención de conocimientos, algunos autores indican que para aprender significativamente y obtener el bien colectivo se deben tomar en cuenta las siguientes variables: el desarrollo de la autonomía moral e intelectual, la capacidad de pensamiento crítico, el autodidactismo, la capacidad de reflexión sobre uno mismo y sobre el propio aprendizaje, la motivación y responsabilidad por el estudio (Díaz Barriga Arceo \& Hernández Rojas, 2002). En este trabajo evaluaremos el aprendizaje significativo a través del pensamiento crítico, motivación y reflexión.

\section{METODOLOGÍA}

La modalidad de la investigación tuvo un enfoque bibliográfico, documental y descriptivo en relación a la tecnología 3D.

Para la creación de recursos didácticos en 3D se aplicó una metodología propuesta en este artículo, que consta de las siguientes fases: Relevamiento de requisitos, diseño 3D, validación de diseño, impresión, ajustes $y$ entrega final, las cuales se describen a continuación:

\section{1) Relevamiento de requisitos}

En esta fase se recogen los requerimientos de la institución, para ello se deberán aplicar las técnicas $e$ instrumentos necesarios que permiten documentar las necesidades educativas que tiene la institución y que deben ser satisfechas en la creación del recurso, se debe definir la asignatura o asignaturas en las que se utilizarán los recursos, características, entre otros aspectos.

\section{2) Diseño 3D}

Para el diseño 3D de cada objeto se determinan las herramientas a usar que para este caso pueden ser: Tinkercad, Solidworks, entre otros. Cada diseño 3D elaborado puede estar en formato $123 \mathrm{dx}$, 3ds, c4d, o mb; el formato que comúnmente se utiliza es el formato $3 \mathrm{ds}$, el cual es un formato que puede ser leído en la mayoría de impresoras existentes.

\section{3) Validacion del Diseño}

En esta fase es necesario realizar pruebas para validar el diseño a través de diferentes herramientas de software lo que nos permite verificar si el producto desarrollado tiene algún defecto y realizar los cambios que sean necesarios.

\section{4) Impresión}

En esta etapa se usa tecnología especializada 
de impresión, lo cual incluye una variedad de herramientas de software disponible para así imprimir el diseño 3D elaborado.

\section{5) Ajustes}

Comprende la mejora continua del prototipo desarrollado, pues en ella se revisa su acabado, se pule el producto y se determina si el producto desarrollado cumple las especificaciones requeridas. En caso de obtener algún tipo de desperfecto se harán los cambios en cualquiera de las fases antes explicadas convirtiéndose el proceso de creación en una oportunidad de mejora del prototipo.

\section{6) Entrega final}

En esta etapa los recursos son evaluados y se determina la aceptación de los mismos. Con ello se procede a la entrega final de los recursos.

\section{$A$. Técnicas de Investigación}

Se usaron además técnicas de investigación como la entrevista y encuesta.

La entrevista consta de 6 preguntas, todas de tipo abierta, y fue aplicada a la Directora de la institución, así se obtuvo. información de las necesidades de recursos didácticos en 3D que requería la institución.

La encuesta usó dos cuestionarios, uno para docentes y otro para estudiantes. El cuestionario para docentes incluyó 7 preguntas. Los cuestionarios pre-test y post-test para los estudiantes constaron de 4 preguntas cada uno.

\section{A. Población y Muestra}

La población de la institución es de 25 estudiantes correspondiente a los cursos: Quinto, sexto y séptimo año de educación básica del Centro Municipal de Apoyo para Personas con discapacidad Visual "Cuatro de Enero" y 3 docentes. Las edades de los niños varían entre los 9 y 11 años de edad.
Debido a que el día en que se aplicó la encuesta no asistieron todos los estudiantes y docentes, sólo se aplicó el instrumento a 15 alumnos y 3 docentes, que equivalen al $64 \%$ de la población total.

\section{DESARROLLO}

Siguiendo la metodología antes descrita, en esta sección se detalla el trabajo desarrollado en cada etapa.

\section{A. Relevamiento de requisitos}

Las necesidades del centro educativo fueron recogidas en una entrevista realizada a la Directora de la institución, en ella se determinaron los requisitos que debían reunir los recursos didácticos a usar, entre ellos: la calidad, su uso, los componentes que se deben incluir cada uno, así como aspectos de color, tamaño, forma y, durabilidad.

En esta fase se documentaron los requisitos específicos y se determinaron los recursos didácticos requeridos para el proceso educativo de estudiantes con discapacidad visual, los cuales se muestran en la tabla 3. 
Tabla 3: Recursos didácticos solicitados por la institución

\begin{tabular}{|l|l|l|}
\hline Recurso didáctico & ¿Para qué sirve? & Área de estudio \\
\hline Cuentos braille en inglés & $\begin{array}{l}\text { Fomenta en los niños un mayor interés en la } \\
\text { lectura y aprendizaje de otros idiomas. }\end{array}$ & Inglés \\
\hline Globo terráqueo & $\begin{array}{l}\text { Apoyo para el aprendizaje de la forma del } \\
\text { mundo y sus continentes. }\end{array}$ & Estudios Sociales \\
\hline Regletas braille & $\begin{array}{l}\text { Motiva a los niños a interesarse más en la } \\
\text { lectura y escritura. }\end{array}$ & Lenguaje \\
\hline $\begin{array}{l}\text { Cuerpos geométricos } \\
\text { (Esferas, cubos) }\end{array}$ & $\begin{array}{l}\text { Permite el aprendizaje de la geometría y el } \\
\text { razonamiento espacial. }\end{array}$ & Matemáticas \\
\hline
\end{tabular}

Fuente: Elaboración propia

\section{B. Diseño 3D}

Cada objeto establecido fue desarrollado usando diversas herramientas de software.

Existen software CAD que son código abierto y gratuito como: Tinkercad y comerciales como: Solidworks, Auto CAD, Blender, entre otros.

En este proyecto, se utilizó Tinkercad para poder realizar el diseño 3D fig.2, porque es de fácil manejo. También se utilizó el software 3D Max 2016, para realizar el diseño 3D de una regleta braille Fig. 3.

Figura 2. Diseño de cuento braille en Tinkercad

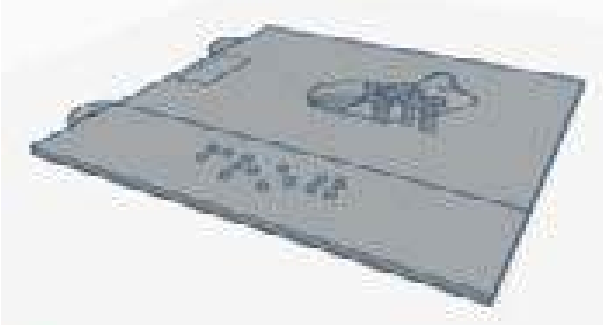

Fuente: Diseño adaptado Basado en

(Clebhsu, 2015)
Figura 3. Diseno de regleta braille

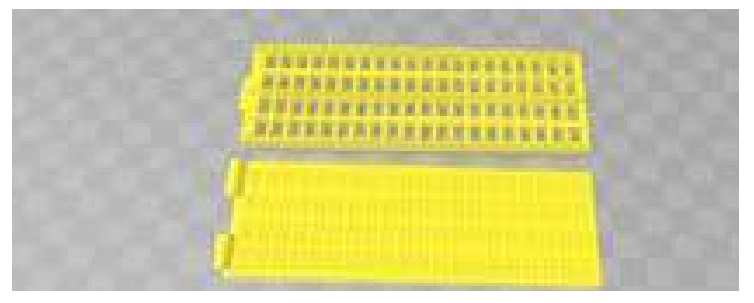

\section{Fuente: Elaboracion propia}

Finalmente, el archivo se envía a la impresora 3D, que procesa la información requerida durante el modelo de impresión 3D.

Para realizar las letras y números en relieve con su significado en braille, utilizamos el sistema de celdas que contienen 6 puntos como se puede ver en la Fig. 4, que representan las celdas que forman el carácter de braille. Estos puntos fueron incorporados al momento de realizar el diseño 3D. 
Figura 4. Dot Braille Tactobook para personas con discapacidad visual

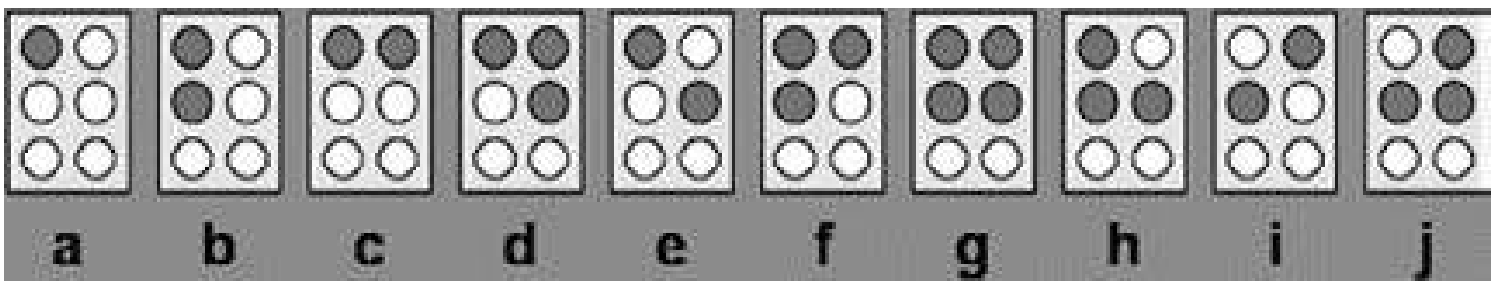

Fuente: Diseño adaptado Basado en (Sutariya, y otros, 2017)

\section{Validación de diseño}

Se revisó el diseño y se detectaron las partes a mejorar, se hicieron los ajustes respectivos.

\section{Impresión}

En este proyecto, se utilizaron las impresoras CoLiDo X3045, Ender 3y Rockstock MAX V3. Estas impresoras se caracterizan por su acabado de gran calidad.

Para poder imprimir en la Ender 3D se utilizó el software CURA; en la impresora Rockstock se usó la herramienta Simplify 3D, mientras que para poder imprimir en la CoLiDo X3045 se utilizó el software Colido, el cual es un software propietario.

A continuación se describen las características y datos técnicos de los recursos 3D por asignatura:

\section{1) RECURSO DE LENGUAJE}

\section{Regleta Braille}

Para la impresión de la regleta braille Fig. 5 y Fig. 6. Se elaboró el diseño en Tinkercad, luego se utilizó la impresora Ender 3D y se aplicaron los ajustes. Los datos técnicos de impresión se observan en la tabla 4.
Figura 5. Base de la regleta braille

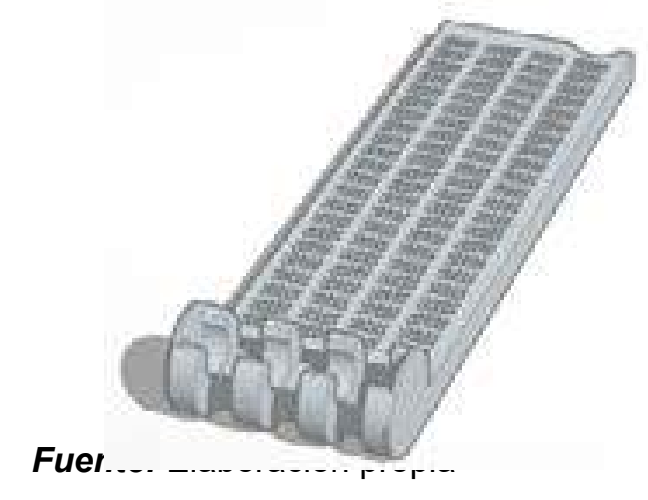

Figura 6. Tapa de la regleta braille

Fuente: Elaboración propia 
Tabla 4: Datos informativos regleta braille

\begin{tabular}{|l|l|l|l|l|l|}
\hline Recursos & Duración & Impresora & Material & $\begin{array}{l}\text { Longitud de } \\
\text { Filamento }\end{array}$ & Peso \\
\hline $\begin{array}{l}\text { Regleta } \\
\text { Braille } \\
\text { (Base) }\end{array}$ & $\begin{array}{l}\text { 2 Horas - 3 Minutos } \\
\text { (Aproximadamente) }\end{array}$ & Ender 3 & PLA & $6050.2 \mathrm{~mm}$ & $18.19 \mathrm{~g}(0.04 \mathrm{lb})$ \\
\hline $\begin{array}{l}\text { Regleta } \\
\text { Braille } \\
\text { (Tapa) }\end{array}$ & $\begin{array}{l}\text { 2 Horas - 22 minutos } \\
\text { (Aproximadamente) }\end{array}$ & Ender 3 & PLA & $5896.2 \mathrm{~mm}$ & $17.73 \mathrm{~g}(0.04 \mathrm{lb})$ \\
\hline
\end{tabular}

Fuente: Elaboración propia

\section{2) RECURSOS DE MATEMÁTICAS}

\section{Cuerpos geométricos (Esfera y Cubo)}

Para la impresión de la esfera y cubo 3D (fig. 7), utilizamos dos impresoras. La esfera fue impresa en la impresora Ender 3D y el cubo en la impresora CoLiDo X3045. En la tabla 5, se pueden observar los datos técnicos de la impresión de cada objeto. Como se puede apreciar el tiempo de impresión de estos objetos es mayor, esto se debe a que se decidió imprimir productos compacto de alta calidad y durabilidad.

Figura 7. Diseño de esfera y cubo en $3 D$
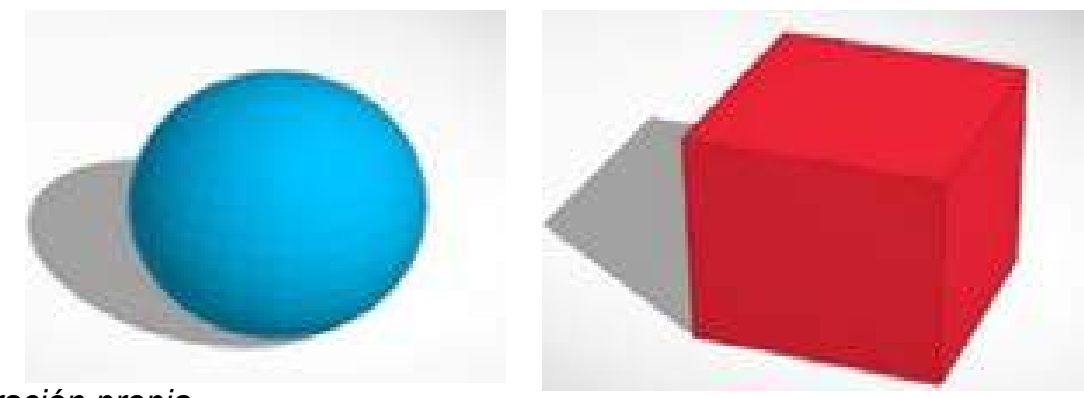

Fuente: Elaboración propia

Tabla 5: Datos informativos del cubo y la esfera

\begin{tabular}{|l|l|l|l|l|l|}
\hline Recursos & Duración & Impresora & Material & $\begin{array}{l}\text { Longitud de } \\
\text { Filamento }\end{array}$ & Peso \\
\hline Cubo & $\begin{array}{l}4 \text { Horas - 44 Minutos } \\
\text { (Aproximadamente) }\end{array}$ & Colido x 3045 & PLA & $27793 \mathrm{~mm}$ & $\begin{array}{l}80,19 \mathrm{~g} \\
(0.17 \mathrm{lb})\end{array}$ \\
\hline Esfera & $\begin{array}{l}8 \text { Horas - 15 Minutos } \\
\text { (Aproximadamente) }\end{array}$ & Ender 3 & PLA & $31607.8 \mathrm{~mm}$ & $\begin{array}{l}95.03 \mathrm{~g} \\
(0.27 \mathrm{lb})\end{array}$ \\
\hline
\end{tabular}

Fuente: Elaboración Propia 


\section{3) RECURSOS DE INGLÉS}

El cuento en relieve y braille es el recurso para la asignatura de Inglés. La presentación del producto es similar a la que se observa en la Fig. 2, pero se realizaron 4 diseños. Los datos técnicos de impresión se observan en la tabla 6 .

Tabla 6: Datos informativos de cuentos en braille

\begin{tabular}{|l|l|l|l|l|l|}
\hline Recursos & Duración & Impresora & Material & $\begin{array}{l}\text { Longitud de } \\
\text { Filamento }\end{array}$ & Peso \\
\hline $\begin{array}{l}\text { Cuentos braille (4 } \\
\text { Diseños) }\end{array}$ & $\begin{array}{l}\text { 2 Hora - 50 Minutos } \\
\text { (Aproximadamente) }\end{array}$ & Ender 3 & PLA & $8701.8 \mathrm{~mm}$ & $\begin{array}{l}26.16 \\
\mathrm{~g}(0.06 \\
\mathrm{lb})\end{array}$ \\
\hline
\end{tabular}

Fuente: Elaboración Propia

\section{4) RECURSOS DE SOCIALES}

Globo Terráqueo.

Para la impresión del globo terráqueo (fig. 8), se

Figura 8. Diseño 3D de un globo terráqueo.

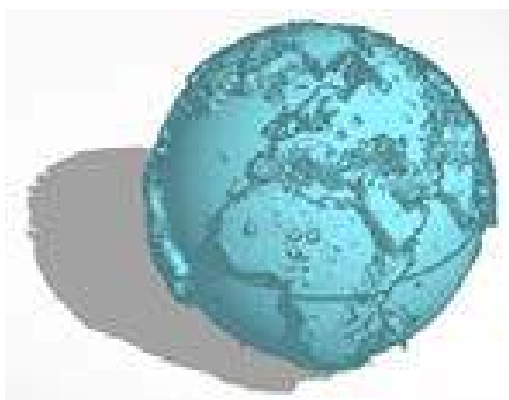

Fuente: (Knape, 2013)

Tabla 7: Datos informativos del globo terráqueo

\begin{tabular}{|l|l|l|l|l|l|}
\hline Recursos & Duración & Impresora & Material & $\begin{array}{l}\text { Longitud de } \\
\text { Filamento }\end{array}$ & Peso \\
\hline Tierra A & $\begin{array}{l}10 \text { Horas }-44 \text { Minutos } \\
\text { (Aproximadamente) }\end{array}$ & Ender 3 & PLA & $41550.2 \mathrm{~mm}$ & $\begin{array}{l}124.93 \mathrm{~g} \\
(0.28 \mathrm{lb})\end{array}$ \\
\hline Tierra B & $\begin{array}{l}12 \text { Horas }-16 \text { Minutos } \\
\text { (Aproximadamente) }\end{array}$ & Ender 3 & PLA & $46555.4 \mathrm{~mm}$ & $\begin{array}{l}139.97 \mathrm{~g} \\
(0.31 \mathrm{lb})\end{array}$ \\
\hline
\end{tabular}

Fuente: Elaboración Propia describen todas las variables de control técnico para la producción del prototipo en la tabla 7 . La impresión se realizó en 2 partes las cuales fueron nombradas como: Tierra A y Tierra B. 


\section{A. Ajustes}

Para cada producto se aplicaron los ajustes necesarios descritos en la metodología propuesta con la finalidad de garantizar un trabajo de calidad. El software usado para los ajustes fue 3D Builder.

\section{B. Entrega final}

Los recursos fueron revisados y evaluados por la Directora de la institución y al definirse su aceptación formal, fueron entregados a los docentes para su uso en las asignaturas de: Matemáticas, Lenguaje, Estudios Sociales e Inglés.

\section{RESULTADOS}

En esta sección se presentan los resultados obtenidos en las encuestas obtenidos en dos instantes: antes del uso de los recursos y después del uso de los mismos en todas las asignaturas establecidas.

\section{1) RESULTADOS PRE-TEST ESTUDIANTES}

En la fig. 9, se puede observar que, de los 15 estudiantes encuestados, el 93\% considera que es importante el uso de los recursos 3D en el aula de clases, criterio similar se aprecia en la fig. 10 , puesto que al $100 \%$ de los alumnos les gustaría hacer uso de los recursos 3D en el aula.

Figura 9. Pre-test estudiantes - Importancia de recursos $3 D$

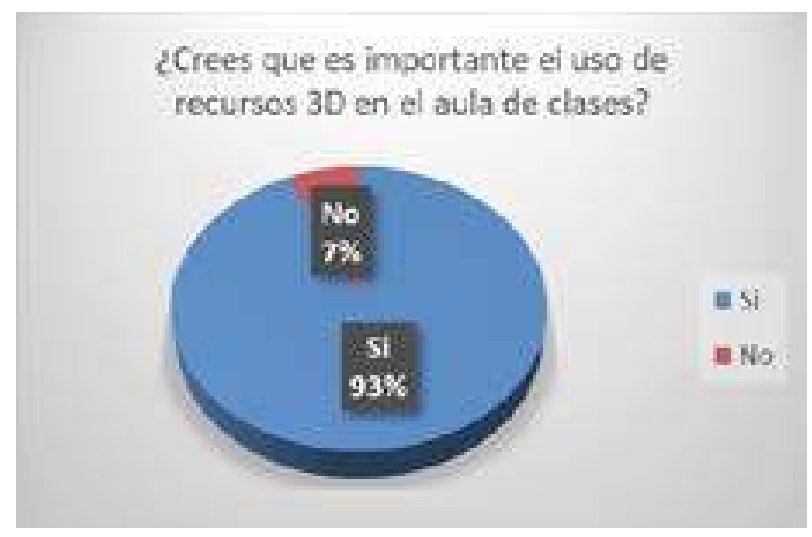

Fuente: Elaboración Propia
Figura 10. Pre-test Estudiantes - Interés en Uso de Recursos 3D

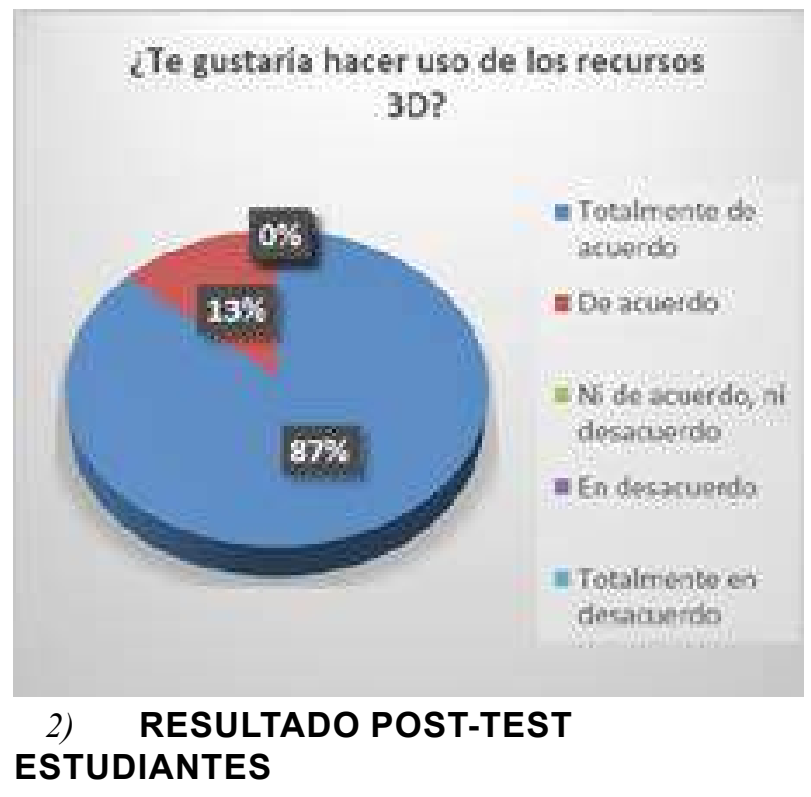

En la fig. 11, se puede notar que luego del uso de los recursos 3D en el aula, el $87 \%$ de los alumnos concluyeron que están totalmente de acuerdo en que el uso de los recursos $3 D$ les ayuda a comprender los temas de estudios, de igual forma el $13 \%$ de estudiantes indicaron estar de acuerdo, lo que destaca el interés general de los estudiantes en los recursos 3D.

Figura 11. Post-test Estudiantes Comprensión de temas de estudio

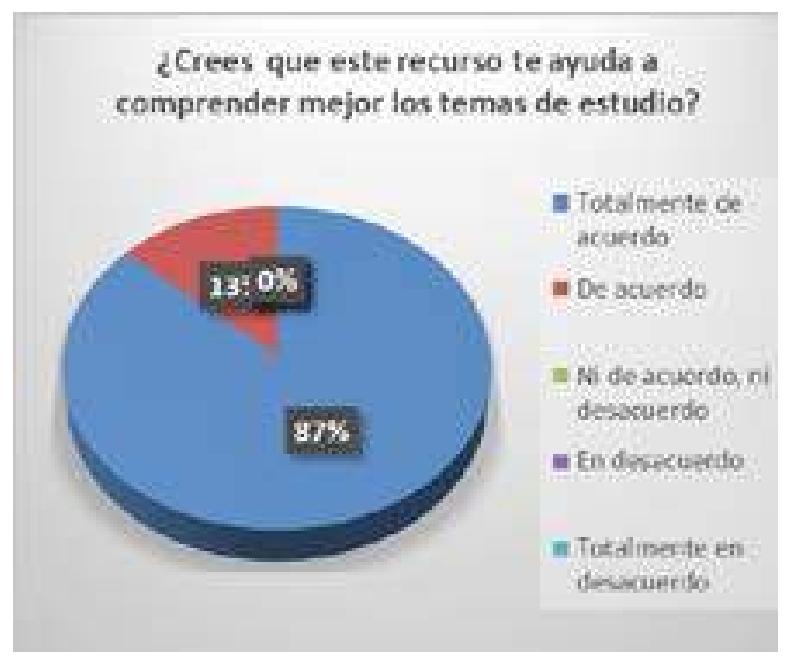

Fuente: Elaboración Propia 


\section{5) RESULTADOS PRE-TEST DOCENTES}

En la fig. 12, se puede observar que el $100 \%$, de los docentes de la institución, consideraron que es necesario el uso de recursos 3D para construir conocimientos en el aula con los alumnos con discapacidad visual.

Figura 12. Pre-test Docentes - Uso de recursos $3 D$ en construcción de conocimientos

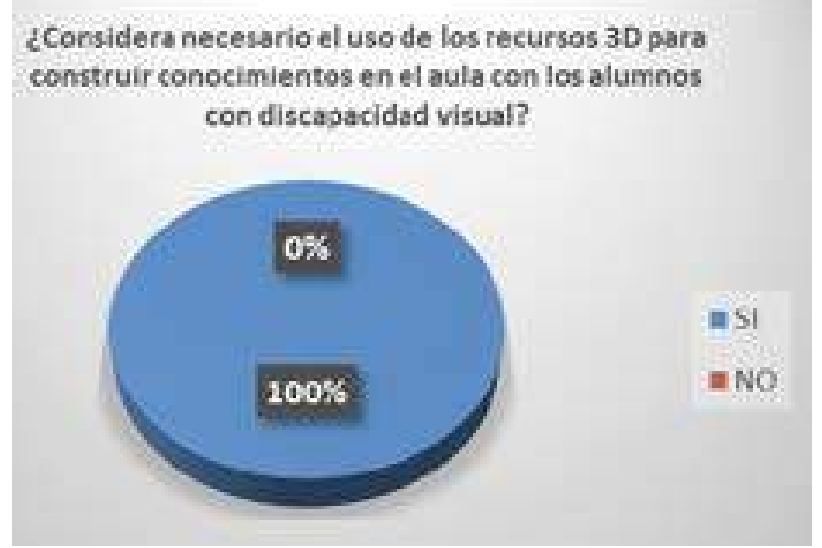

Fuente: Elaboración Propia

En la fig. 13 , el $71 \%$ de los docentes concluyeron que están totalmente de acuerdo y el $29 \%$ de acuerdo, en que los recursos $3 \mathrm{D}$ son un medio que facilitaría una mejor compresión de los temas de su asignatura. Ninguno de los docentes estuvo en desacuerdo en esta interrogante.

Figura 13. Pre-test Docentes - Facilitar comprensión de temas

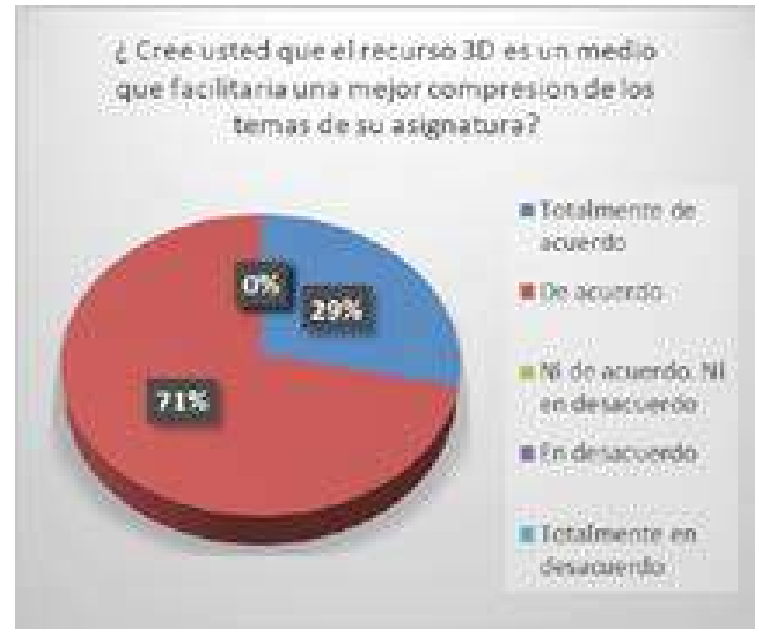

Fuente: Elaboración Propia
Respecto a si los recursos didácticos en 3D facilitarían la evaluación del proceso de aprendizaje de los estudiantes, el 100\% manifestó estar de acuerdo en esa pregunta.

\section{6) POST-TEST DOCENTES}

En la fig. 14, luego del uso de los recursos 3D en el aula, el $71 \%$ de los docentes encuestados, respondieron que están de acuerdo, en que el uso de los recursos 3D motivan a los estudiantes en su proceso de aprendizaje, un $29 \%$ de los docentes respondieron que están totalmente de acuerdo.

Figura 14. Post-test Docentes - Motivación en el proceso de aprendizaje

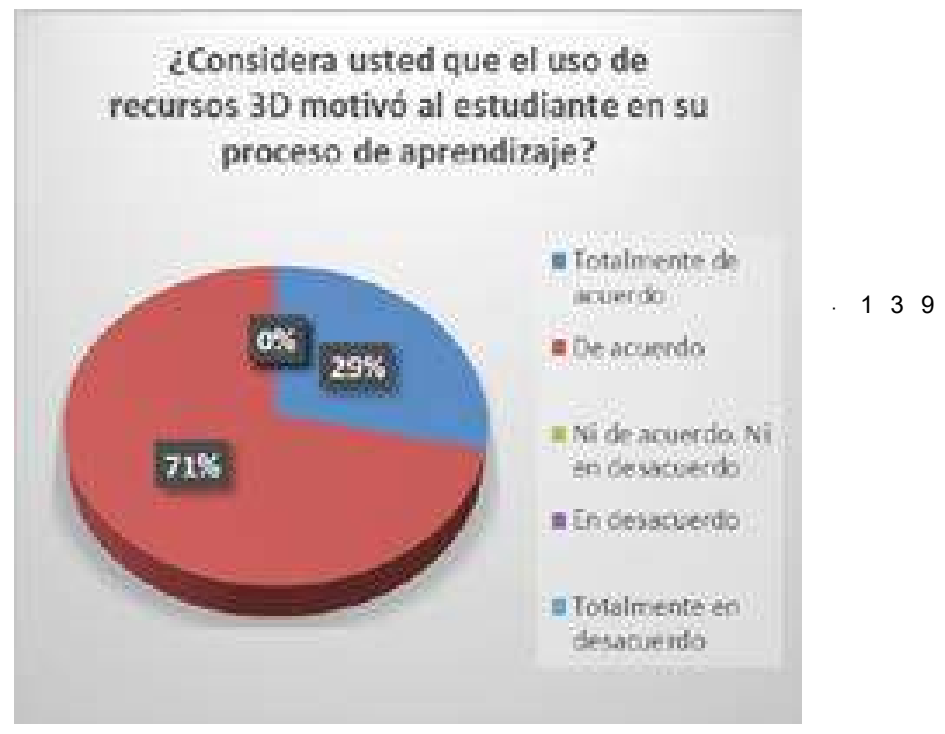

\section{Fuente: Elaboración Propia}

En la fig. 15, se observan los resultados obtenidos en las variables analizadas: motivación, pensamiento crítico, desarrollo de la inteligencia espacial, aprendizaje significativo, reflexión y evaluación del proceso de aprendizaje. Siendo el aprendizaje significativo la opción con mayor porcentaje, la cual se complementa con las variables pensamiento crítico, motivación, reflexión, que en conjunto suman un $82 \%$. 
Figura 15. Post-test Docentes - Variables objeto de análisis

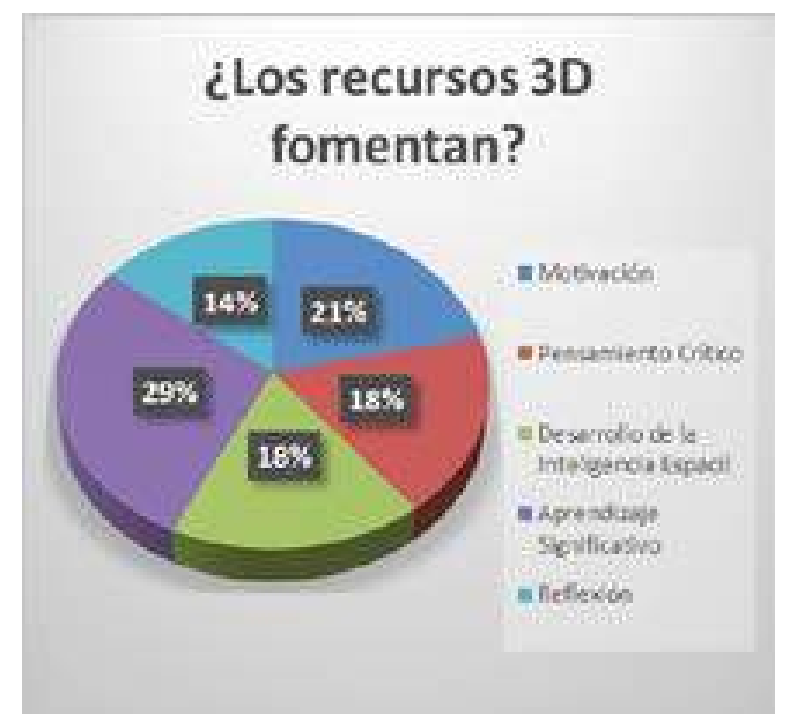

Fuente: Elaboración Propia

\section{DISCUSIÓN}

En la búsqueda de información para este proyecto, encontramos trabajos elaborados previamente, en los cuales se han realizado recursos didácticos para su uso en el aula, pero elaborados a base de papel (J. R. Lazzarin and e. S. Tiago Stefanelo, 2017) u otros materiales (F. García Soria \& P. Ruiz Prieto, 2010), recursos que se deterioran con el uso y el tiempo, a diferencia de los recursos impresos en 3D.

Entre todas las ventajas que se analizaron de los recursos didácticos impresos en $3 \mathrm{D}$, se determinó que las mejores fueron: Durabilidad con un porcentaje de $29 \%$; facilidad de uso, $28 \%$ y resistencia/consistencia con un $24 \%$.

Los resultados de las encuestas tanto de docentes y estudiantes coinciden en que los recursos impresos en 3D, fomentan el proceso de aprendizaje significativo, despiertan interés y motivación en los estudiantes en relación con los materiales tradicionales usados para el diseño de recursos didácticos como fomix y cartón. La desventaja es que no todas las instituciones pueden acceder a los equipos tecnológicos para crear este tipo de recursos impresos en 3D por el alto costo de los mismos.

\section{CONCLUSIONES}

La metodología establecida para la creación de recursos didácticos en 3D permitió generar recursos didácticos para su uso por estudiantes con discapacidad visual de una unidad educativa especial. Los productos fueron desarrollados atendiendo de forma personalizada las necesidades de docentes y estudiantes de la institución.

Los recursos $3 \mathrm{D}$ que incluyeron el sistema braille despertaron mayor interés en los estudiantes respecto a los que no lo usaron; a través del sentido del tacto los $\mathrm{EcD}$ apreciaron objetos y conocieron el nombre de los mismos o desarrollaron lecturas en el sistema de lectoescritura braille.

Los recursos didácticos en 3D permitieron favorecer el aprendizaje significativo de los niños con discapacidad visual, lo que fue corroborado por los docentes a través de las actividades desarrolladas. Las variables que evidencian el aprendizaje significativo como pensamiento crítico, motivación y reflexión fueron evaluadas. Este tipo de recursos permitió además desarrollar un proceso de aprendizaje activo, participativo e inclusivo.

A través de los recursos didácticos 3D, los estudiantes hacen uso de la inteligencia espacial y representativa, lo que le sirve de apoyo al docente para facilitar a sus estudiantes, la exploración táctil e interiorización del tema que se está desarrollando en la clase.

Se recomienda implementar este tipo de recursos didácticos en instituciones regulares que incluyen estudiantes con discapacidad visual ya que favorecería al aprendizaje de todos y a su vez se estaría fomentando un entorno de inclusión educativa. 


\section{AGRADECIMIENTOS}

A la Universidad Politécnica Salesiana, al Grupo de Innovación Educativa GIE-IDI por permitirnos colaborar en el proyecto PRO3D (Prototipado de recursos didácticos en $3 \mathrm{D}$ ), a la Psicóloga Geoconda del Rocío Soledispa Reyes, Directora del Centro Municipal de Apoyo para personas con discapacidad visual "Cuatro de Enero", por todas las facilidades brindadas para el desarrollo del trabajo y a los docentes que participaron en el proceso, al CAI (Centro de Apoyo para la inclusión), y a la tifloteca de la UPS Sede Guayaquil por el préstamo de la tecnología en 3D para hacer realidad este proyecto.

\section{REFERENCIAS BIBLIOGRÁFICAS}

Arnaiz, P. (2012). Escuelas eficaces e inclusivas: cómo favorecer su desarrollo. Education Siglo XXI, vol. 30(no. 1), pp. 44.

Castro, S., Casar, L., \& García, A. (2018). Reflexiones sobre la enseñanza inclusiva del inglés apoyada por tecnologías. Recuperado el 30 de Julio de 2019, de http://scielo.sld.cu/pdf/rces/ v38n1/0257-4314-rces-38-01-e12.pdf

Chancusig Chisag, J. C., Flores Lagla, G. A., Venegas Alvarez, G. S., Cadena Moreano, J. A., Guaypatin Pico, O. A., \& Izurieta Chicaiza, E. M. (12 de octubre de 2017). Utilización de recursos didácticos interactivos a través de las TIC'S en el proceso de enseñanza aprendizaje en el área de matemática. Revista Boletín Redipe, 6(4), 112-134. Recuperado el 23 de enero de 2020 , de https://revista. redipe.org/index.php/1/article/view/229

Clebhsu. (1 de Nov de 2015). Thingiverse.com. Obtenido de https://www.thingiverse. com/thing:1104933
Cruz Verdugo, A. (30 de abril de 2016). Clasificación de triángulos de acuerdo a la longitud de sus lados: una propuesta para la enseñanza y aprendizaje en geometría. Revista Boletín Redipe, 5(4), 42-49. Recuperado el 23 de enero de 2020, de https://revista.redipe.org/ index.php/1/article/view/89

Díaz Barriga Arceo, F., \& Hernández Rojas, G. (2002). Estrategias Docentes para un Aprendizaje Significativo Una interpretación constructiva. En F. Díaz Barriga Arceo, \& G. Hernández Rojas, Estrategias Docentes para un Aprendizaje Significativo Una interpretación constructiva ( $3^{\text {a }}$ edición ed., pág. 405). México: Mc Graw Hill.

García Soria F., \& Ruiz Prieto P. (2010). Mapas geográficos para personas ciegas $y$ deficientes visuales. Integración Revista sobre Discapacidad Visual, 4(57), 5658. Recuperado el 23 de enero de 2020, de https://www.once.es/dejanosayudarte/la-discapacidad-visual/revistaintegracion/2010-integracion-56-58/ numero-57/57-04-garcia-ruiz-mapasgeograficos-para-personas.pdf/view

Gamboa, M., Briceño, J., \& Camacho, J. (2015). "Caracterización de estilos de aprendizaje y canales de percepción de estudiantes universitarios". Universidad del Zulia Repositorio Digital(no. 3), pp. 509-527.

Horizon Report. (2016). Resumen informe Horizon 2011-2016. Recuperado el 30 de Julio de 2019, de http://www.ite. educacion.es 
Horne, R., \& Hausman, K. K. (2014). Defining aditive manufacturing. En $3 D$ Printing for Dummies (pág. pp. 375). Cánada. Obtenido de https://bibliotecas. ups.edu.ec:2708/lib/upsal/detail. action?docID=1609155.

Lazzarin J. and Stefanelo T. (2017). "Matemática inclusiva: Ensinando matrizes a deficientes visuais". Ciência e Natura, vol. 39(no. 1), pp. 128-126. Obtenido de https://bibliotecas.ups.edu.ec:2598/ docview $/ 1872777692$ ?accountid=32861

Knape. (28 de Oct de 2013). Thingiverse. Recuperado el 31 de Julio de 2019, de https://www.thingiverse.com/ thing: 173130

Kway, E. H., Mohd Salleh, N., \& Abd. Majid, R. (2010). Slate and Stylus: An alternative tool for braille writing. Procedia - Social and Behavioral Sciences, vol. 7, pp. 326-335. Obtenido de https://doi. org/10.1016/j.sbspro.2010.10.045

Lucía, C. (14 de Enero de 2019). Reporte Gartner 2019: La impresión 4D comienza su ascenso. Recuperado el Julio de 2019, de 3D natives: El sitio web de la impresión 3D: https:// www.3dnatives.com/es/gartner-2019impresion-4d-140120192/

Madrid, V. A. (2017). Introducción. En A. M. (Ingeniero), Tecnología de la impresion 3D: la fábrica del futuro (pág. 200). Madrid: Editor Antonio Madrid Vicente.

Mercadé, A. (s.f.). Los 8 tipos de inteligencia según Howard Gardner: a teoría de las inteligencias múltiples. Recuperado el Julio de 2019

Ministerio de Educación del Ecuador. (2018). Ministerio de Educación Escuelas Inclusivas. Recuperado el 20 de Enero de 2020, de https://educacion.gob.ec/ escuelas-inclusivas/

Proyecto Malala - Inteligencia Visual-Espacial. (s.f.). Recuperado el 30 de Julio de 2019, de Proyecto Malala - Inteligencia Visual-Espacial: http://proyectomalala. beecocentric.com/?page_id=1616

República del Ecuador. (25 de Septiembre de 2012). Ley Orgánica de Discapacidades Asamblea Nacional. Recuperado el 30 de Julio de 2019, de https://www. consejodiscapacidades.gob.ec/wpcontent/uploads/downloads/2014/02/ ley_organica_discapacidades.pdf

República del Ecuador. (20 de Octubre de 2018). Constitución de la República del Ecuador. Montecristi, Ecuador. Recuperado el 30 de Julio de 2019, de https://www.asambleanacional.gob. ec/sites/default/files/documents/old/ constitucion_de_bolsillo.pdf

Rúa, R., Blasnilo, E., Jiménez D, F., Gutiérrez A., G. A., \& Villamizar, N. I. (2018). Impresión 3D como herramienta didáctica para la enseñanza de algunos conceptos de ingeniería y diseño. Ingeniería, vol. 23(no. 1), pp. 70 - 83.

Salguero Barba, N. G., \& García, C. P. (may de 2017). Autoestima, educación emocional y su incidencia en el proceso de enseñanza aprendizaje de los estudiantes en las instituciones educativas. Revista Boletín Redipe, 6(5), 84 - 92. Obtenido de https://revista. redipe.org/index.php/1/article/view/261 
Sutariya, Raj \& Singh, Himanshu \& Babariya, Sudhir \& Kadiyar, Sajid \& Modi, \& Darshan. (2017). Refreshable braille display for the visually impaired. 2017 14th IEEE India Council International Conference (INDICON) (págs. 1 - 5). Roorkee. doi:10.1109/ INDICON.2017.8487232

Tomalá Cabezas, L., \& Naranjo Sanchéz, B. (2019). "Sistemas de seguimiento académico a estudiantes con discapacidad en las universidades de Guayaquil". Uniandes Episteme. Revista digital de Ciencia, Tecnología e Innovación, vol. 6(no. 1), pp. 124-37.

Umair, M., \& Kim, W. S. (2015). An online 3D printing portal for general and medical fields. 2015 International Conference on Computational Intelligence and Communication Networks (CICN), (págs. pp. 278 - 282). Jabalpur. doi:10.1109/CICN.2015.62

Universidad de Las Palmas de Gran Canaria. (s.f.). Desarrollo psicológico del niño ciego. Recuperado el 30 de Julio de 2019, de https://www2.ulpgc.es/hege/ almacen/download/1/1767/capitulo7.pdf 